\title{
Avaliação e tratamento fisioterápico na doença arterial obstrutiva periférica de membro superior: um estudo de caso
}

\author{
Assessment and physical therapy treatment for peripheral artery \\ occlusive disease of the upper limb: a case study

\begin{abstract}
Danielle Aparecida Gomes Pereira ${ }^{1}$, Marcelle Xavier Custódio ${ }^{1}$, João Paulo Ferreira de Carvalho ${ }^{1}$, André Maurício Borges de Carvalho ${ }^{2}$, Inácio Teixeira da Cunha-Filho ${ }^{3}$
\end{abstract}

\begin{abstract}
Resumo
O objetivo deste artigo é apresentar um estudo de caso em que se propõe um protocolo de avaliação e intervenção para uma paciente com claudicação de membro superior. Descrição do caso: mulher de 50 anos com 4 meses de evolução de quadro de obstrução de artéria braquial esquerda pós-cateterismo. Na avaliação com Doppler contínuo, observou-se presença de som monofásico em artérias radial e ulnar. No teste do cicloergômetro, a dor isquêmica iniciou aos 2 minutos e 30 segundos e atingiu o ponto máximo aos 9 minutos e 26 segundos. Foi realizado tratamento em cicloergômetro três vezes por semana durante 8 semanas. Após o tratamento, o tempo de teste em cicloergômetro aumentou: dor inicial aos 5 minutos e 7 segundos e máxima aos 18 minutos. A paciente relatou desaparecimento da cianose e melhora na realização de atividades de vida diária. $\mathrm{O}$ protocolo de avaliação proposto envolvendo medidas objetivas (cicloergômetro) e subjetivas (questionário SF-36 traduzido e validado em português) foi bem tolerado, tendo sido capaz de detectar alterações no estado funcional da paciente. As alterações detectadas no tempo de surgimento de dor inicial e de dor máxima podem ter acontecido de modo espontâneo, mas não se pode descartar que a intervenção possa, potencialmente, ser benéfica para indivíduos com claudicação de membros superiores. Os resultados observados neste estudo de caso avalizam futuros estudos envolvendo maior número de participantes.
\end{abstract}

Palavras-chave: Arteriopatia oclusiva, fisioterapia, membros superiores.

\section{Introdução}

A doença arterial obstrutiva periférica (DAOP) é caracterizada por perfusão inadequada no membro, usualmente devido a processo aterosclerótico ${ }^{1}$. Os principais sinais e sintomas são: dor, redução de temperatura

\begin{abstract}
The aim of this paper was to present a case study proposing a protocol for assessment and rehabilitation of a patient with upper limb intermittent claudication. Case description: 50-year-old woman with obstruction of the left brachial artery secondary to catheterization performed 4 months ago. Monophasic sound was observed during continuous Doppler ultrasound assessment of both the radial and ulnar arteries. During the arm crank test, ischemic pain started at 2 minutes and 30 seconds of cranking, while maximal pain was reached at 9 minutes and 26 seconds. The patient was treated by arm cranking exercises performed three times a week for 8 weeks. After the treatment, arm crank time increased: ischemic pain onset was at 5 minutes and 7 seconds and maximal pain was reached at 18 minutes. The patient reported disappearance of cyanosis and improvement in performance of daily activities. The assessment protocol comprehended both subjective (validated Brazilian Portuguese version of SF-36 questionnaire) and objective (arm crank) measurements and was well tolerated, besides being able to detect changes in the patient's functional capacity. Changes detected at pain onset and at maximal pain may have occurred spontaneously, but it cannot be ruled out that this intervention can be potentially beneficial for individuals with upper limb claudication. The results observed in this case study warrant further studies involving larger sample size.
\end{abstract}

Keywords: Arterial occlusive disease, physical therapy, upper limb.

cutânea, ausência ou diminuição dos pulsos do membro acometido, cianose, hipoperfusão, alterações tróficas e claudicação intermitente, sendo este último o sintoma mais freqüentemente observado nos membros inferiores ${ }^{1}$. Nos membros superiores, o sintoma claudicante pode ocorrer principalmente quando a atividade

1. Fisioterapeuta, Centro Universitário de Belo Horizonte (UNI-BH), Belo Horizonte, MG.

2. Médico angiologista, UNI-BH, Belo Horizonte, MG.

3. PhD. Fisioterapeuta, UNI-BH, Belo Horizonte, MG.

Apresentado sob a forma de pôster no Congresso de Angiologia e Cirurgia Vascular, em Juiz de Fora (MG), em junho de 2004.

Artigo submetido em 26.07.07, aceito em 14.01.08.

J Vasc Bras. 2008;7(1):72-75.

Copyright $\odot 2008$ by Sociedade Brasileira de Angiologia e de Cirurgia Vascular 
é realizada com os braços em elevação $0^{2-4}$. A DAOP nos membros superiores tem menor incidência e está mais freqüentemente associada a iatrogenia decorrente de cateterismo ${ }^{2,3}$.

Dentre as formas de tratamento para DAOP têm-se intervenção cirúrgica, terapia medicamentosa e programa de reabilitação fisioterápica como alternativas para a redução dos sintomas claudicantes, melhora da capacidade funcional, prevenção da progressão da oclusão vascular e das complicações cardiovasculares ${ }^{5}$. Entretanto, não existem protocolos específicos para a avaliação funcional de claudicação de membros superiores, nem mesmo protocolo específico para a reabilitação de tais pacientes, devido, provavelmente, à baixa incidência/prevalência da claudicação de membro superior. Apesar da presença da claudicação e da limitação funcional observadas nessa condição, os protocolos de avaliação e tratamento ainda não são sistematizados. O objetivo deste artigo é, portanto, apresentar um estudo de caso em que se propõe um protocolo de avaliação e intervenção de uma paciente com claudicação de membro superior.

\section{Descrição do caso}

Mulher de 51 anos, portadora de DAOP no membro superior esquerdo, secundária a obstrução da artéria braquial após cateterismo. A paciente apresentava 4 meses de evolução do quadro desde a realização do cateterismo, com piora progressiva de sintomas, culminando no afastamento do trabalho devido ao sintoma claudicante que lhe impunha restrições funcionais.

As queixas incluíam dor limitante para a realização de tarefas do lar (classificada como estágio II na classificação de Fontaine adaptada para membros superiores: aparecimento de dor isquêmica durante esforços físicos ${ }^{4}$ ), parestesia e perda de força no membro superior esquerdo. A paciente era hipertensa, controlada por terapia medicamentosa, e tinha antecedentes de distúrbio de coagulação. Os medicamentos Diovan ${ }^{\circledR}(80 \mathrm{mg})$ qd, Angipress ${ }^{\circledR}$ (25 mg) qd e ácido acetilsalicílico (500 $\mathrm{mg}$ ) qd estavam em uso.

No exame físico, observou-se membro superior esquerdo cianótico, baixa temperatura cutânea e ausência de pulso radial à palpação. Na avaliação ultrasonográfica (Doppler contínuo), observou-se presença de fluxo monofásico em artérias radial e ulnar.

\section{Testes utilizados}

\section{- Teste em cicloergômetro de membros superiores}

(CEMS). Teste de esforço progressivo iniciado com carga de 15 Watts, com incrementos de 5 Watts a cada 3 minutos até atingir 12 minutos, e de 10 Watts a partir de 12 minutos até o alcance de dor isquêmica máxima. Foram registrados o tempo de início da dor e o tempo durante o qual a paciente foi capaz de manter a atividade. Após o teste, foi registrado o tempo necessário para cessar a dor no membro superior esquerdo, denominado tempo de recuperação ${ }^{6}$.

- Avaliação da qualidade de vida. Foi utilizado o questionário SF-36, em sua versão traduzida para português e validada por Ciconelli et al. ${ }^{7}$. O SF-36 é um instrumento genérico de avaliação de qualidade de vida, multidimensional, composto por 36 itens que avaliam oito domínios: capacidade funcional, aspecto físico, dor, estado geral de saúde, vitalidade, aspectos social e emocional e saúde mental. Apresenta uma pontuação de 0 a 100 , sendo 0 o pior estado geral de saúde e 100 o melhor ${ }^{7}$.

Neste estudo de caso, foram cumpridos os termos da Resolução 196, de 10 de outubro de 1996, do Conselho Nacional de Saúde. Todos os testes foram aplicados antes e após a intervenção com consentimento livre e esclarecido da paciente. A intervenção fisioterapêutica teve uma freqüência de três vezes por semana e estendeu-se por 8 semanas, consistindo de programa ambulatorial com alongamento de musculatura da cintura escapular, bíceps braquial e flexores dos punhos, e condicionamento em CEMS até atingir dor máxima. $\mathrm{O}$ treinamento foi iniciado com 15 Watts, evoluindo gradativamente para 35 Watts. A paciente deveria completar 20 minutos de treinamento na bicicleta independentemente dos períodos em que precisava descansar. Também foi realizado programa domiciliar, em que a paciente deveria insistir em suas atividades de vida diária até que a dor máxima fosse alcançada. Só então a paciente deveria interromper a atividade, permitindo que o sintoma fosse aliviado. Tão logo a dor permitisse, a atividade deveria ser retomada.

\section{Resultados}

Não foram observadas diferenças entre as variáveis hemodinâmicas obtidas com o CEMS nos testes pré e 
Tabela 1 - Valores obtidos no teste em cicloergômetro de membros superiores

\begin{tabular}{lcc}
\hline & Pré-tratamento & Pós-tratamento \\
\hline FC inicial (bpm) & 68 & 69 \\
FC final (bpm) & 79 & 79 \\
PA inicial (mmHg) & $110 / 80$ & $100 / 80$ \\
PA final (mmHg) & $130 / 80$ & $120 / 80$ \\
Início da dor (s) & 150 & 307 \\
Dor máxima (s) & 566 & 1080 \\
Tempo recuperação (s) & 167 & 161 \\
Carga (Watts) & 30 & 50 \\
\hline
\end{tabular}

$\mathrm{FC}=$ freqüência cardíaca; $\mathrm{PA}=$ pressão arterial.

pós-tratamento. Entretanto, a carga final aumentou de 30 Watts para 50 Watts, perfazendo um aumento de $66 \%$. Não houve diferença relevante entre o tempo de recuperação ao final do teste antes e após o tratamento. $\mathrm{O}$ tempo para surgimento da dor inicial aumentou em $105 \%$, enquanto o tempo para surgimento da dor máxima aumentou em $81 \%$ (Tabela 1). A avaliação da qualidade de vida apresentou índices que indicam melhora, sobretudo em relação aos aspectos social e emocional do SF-36, que atingiram 100\% (Tabela 2).

\section{Discussão}

O presente estudo teve por objetivo apresentar um protocolo de avaliação e tratamento de claudicação de membro superior. Tanto o processo de avaliação quanto o tratamento proposto demonstraram ser exeqüíveis e bem tolerados pela paciente. $\mathrm{O}$ protocolo de avaliação foi capaz de detectar mudanças funcionais e na qualidade de vida no período em que a paciente esteve em tratamento.

Não foram observadas alterações substanciais nas variáveis hemodinâmicas, já que o uso do betabloqueador leva a resposta atenuada tanto de freqüência quanto de pressão arterial. A proporção de melhora observada com o teste CEMS foi clinicamente relevante. Isso talvez se explique pela especificidade da tarefa realizada, ou seja, pela semelhança entre a maneira como foi realizado o condicionamento no tratamento e a forma como o teste foi realizado.

A paciente também relatou abolição de dor durante atividades de vida diária. Entretanto, essa alteração na percepção não foi detectada totalmente pelo SF-36, já que o escore pra o item dor alcançou $62 \%$, e não $100 \%$.

Tabela 2 - Valores obtidos no questionário SF-36

\begin{tabular}{lcc}
\hline Itens & Pré-tratamento (\%) & Pós-tratamento (\%) \\
\hline Capacidade funcional & 65 & 95 \\
Aspecto físico & 0 & 75 \\
Dor & 22 & 62 \\
Estado geral de saúde & 67 & 82 \\
Vitalidade & - & 95 \\
Aspecto social & 37,5 & 100 \\
Aspecto emocional & 0 & 100 \\
Saúde mental & - & 96 \\
\hline
\end{tabular}


Isso sugere que a compreensão do SF-36 pode não ser satisfatória para a avaliação do paciente com doença arterial oclusiva, ainda que o teste tenha sido validado para a população brasileira ${ }^{7}$.

Tem sido sugerido que a isquemia de membro superior causada por oclusão arterial aguda decorrente de iatrogenia, como a observada neste estudo, pode apresentar melhora clínica espontânea e compensação do quadro isquêmico. Entretanto, o que se observou nesta paciente foi um progressivo agravamento do seu quadro clínico e funcional no período decorrido de 4 meses desde a instalação da obstrução até seu encaminhamento para tratamento fisioterapêutico. A paciente esteve impedida de executar suas tarefas usuais devido ao sintoma isquêmico e, desta forma, à semelhança do tratamento utilizado para sintoma isquêmico de membros inferiores decorrente de obstrução aterosclerótica, foi proposta uma intervenção através de atividade física programada.

A paciente foi submetida a um regime de exercício físico supervisionado, com intensidade tal que o limiar de dor claudicante fosse atingido. A intervenção foi mantida por um período de 8 semanas, com uma freqüência de três vezes por semana. Além disso, a paciente foi instruída a executar as tarefas de vida diária, a despeito do surgimento do sintoma. No caso de surgimento do sintoma claudicante durante as atividades no lar, a paciente foi instruída a interrompê-las até que a dor aliviasse, retomando então sua execução. Esse procedimento é semelhante ao que é proposto durante o exercício supervisionado. Dessa forma, ainda que seja mais freqüente que a melhora no status clínico e funcional dos pacientes com lesão iatrogênica do leito arterial ocorra de modo espontâneo, não se deve descartar completamente a possibilidade de a forma de intervenção proposta ter contribuído para o arrefecimento dos sintomas e para a melhora na capacidade funcional. Contudo, é necessário que futuros estudos, incluindo grupo controle e maior número de participantes, sejam realizados para determinar a eficácia da intervenção proposta, uma vez que o protocolo proposto demonstra sensibilidade para mudanças funcionais, sejam elas espontâneas ou não.

\section{Conclusão}

O protocolo de avaliação proposto, envolvendo medidas objetivas (cicloergômetro) e subjetivas (SF-36), foi bem tolerado, tendo sido capaz de detectar alterações no estado funcional da paciente. É possível que as alterações detectadas pelo protocolo proposto tenham ocorrido espontaneamente. No entanto, não devemos descartar que a intervenção possa, potencialmente, contribuir para a mitigação de sintomas e a restauração da capacidade funcional. Os resultados observados neste estudo de caso avalizam futuros estudos envolvendo maior número de participantes.

\section{Referências}

1. Maffei FHA, Lastória S, Yoshida WB, Rollo HA. Diagnóstico clínico das doenças arteriais periféricas. In: Maffei FHA, Lastória S, Yoshida WB, Rollo HA, editors. Doenças vasculares periféricas. Rio de Janeiro: Medsi, 2002. p. 287-304.

2. Gornik HL, Beckman JA. Peripheral arterial disease. Circulation. 2005;111:e169-72.

3. Nakano L, Wolosker N, Rosoki RA, Netto BM, Puech-Leão P. Objective evaluation of upper limb claudication: use of isokinetic dynamometry. Clinics. 2006;61:189-96.

4. Guirov K, Stoyanov K, Topalov I. New method and device for assessment of functional capacity of upper extremity with chronic ischemia. Int Angiol. 1997;16:245-9.

5. Schmieder F, Comerota AJ. Intermittent claudication: magnitude of the problem, patient evaluation, and therapeutic strategies. Am J Cardiol. 2001;87:3D-13D.

6. Outras condições clínicas que influenciam a prescrição do exercício. In: American College of Sports Medicine. Diretrizes do ACSM para testes de esforço e sua prescrição. Rio de Janeiro: Guanabara Koogan; 2000. p. 136-42.

7. Ciconelli RM, Ferraz MB, Santos W, Meinão I, Quaresma MR. Tradução para a língua portuguesa e validação do questionário genérico de avaliação de qualidade de vida SF-36 (Brasil SF-36). Rev Bras Reumatol. 1999;39:143-50.

Correspondência:

Danielle Aparecida Gomes Pereira

Rua João Gualberto Filho, 1260/604, Bairro Sagrada Família

CEP 31035-570 - Belo Horizonte, MG

Tel.: (31) 3309.9137, (31) 9103.7415

E-mail: d.fisio@ig.com.br 